\title{
Comparative Study between Arthroscopic and Mini Open Rotator Cuff Repair
}

\author{
AHMED KHOLEIF, M.D.*; SHERIF A. KHALED, M.D.*; AHMED RIZK, M.D.*; EBEED YASIN, M.D.*; \\ AMR F. HAFEZ, M.Sc.**; YOUSEF M. AHMED, M.Sc.***; RAED A. ABDALLAH, M.Sc.*; \\ REDA A. SHETA, M.Sc.* and AHMED I. NAWWAR, M.Sc.*
}

The Department of Orthopedic Surgery, Faculty of Medicine, Cairo University*, Police Hospital Agouza** and El-Haram Hospital***

\begin{abstract}
Background: Tears of the rotator cuff are among the most frequently encountered causes of pain and dysfunction in the shoulder. The mechanism of injury may be traumatic or degenerative. Operative treatment includes open, mini open, arthroscopic techniques. We hypothesized a comparative study between mini open and arthroscopic rotator cuff repair.

Aim of Work: To assess the functional outcome of arthroscopic rotator cuff repair and mini-open rotator cuff repair with ASES scores.

Methods: A prospective analysis of 30 patients with rotator cuff tears were evaluated before and after repair of the cuff tear by the arthroscopic repair technique (Group A) and the mini-open repair technique (Group B). The first case was managed in September 2014 and the last in June, 2016. All the cases were managed in El-Kasr Al-Ainy and PoliceHospital Agouza. The follow-up period was 6 months. The patients were evaluated using the American Shoulder \& Elbow Society score (ASES), both pre-operatively and postoperatively.

Results: After follow-up at 3 months ASES total score (100 points) $>90$ was no patients for mini-open group and for arthroscopic group was 3 patients (20\%) after 6 months was 12 patients $(80 \%)$ for mini-open and 13 patients $(86.6 \%)$.

Conclusion: Mini-open and arthroscopic rotator cuff repair both improved the function of patients, who failed nonoperative treatment, but there is no statistical or clinically important difference in function between the two surgical approaches according to ASES score at 6 months postoperatively but arthroscopic earlier improvement in pain and functional out come.
\end{abstract}

Key Words: Rotator cuff-Arthroscopic-Mini-open.

\section{Introduction}

ROTATOR cuff tears are among the most frequently encountered causes of pain and dysfunction in the shoulder [1]. Rotator tears can be secondary

Correspondence to: Dr. Ahmed Kholeif, The Department of Orthopedic Surgery, Faculty of Medicine, Cairo University to various etiologies: First of all impingement syndrome and then instability or trauma [2]. In all clinical reports, the incidence of cuff defects is relatively low before the age of 40 years, begins to rise in the 50-to 60-year-old age group, and continues to increase in the 70 years and older age group [3]. Rotator cuff pathology may be graded arthroscopically using the "ABC" system in which " $A$ " represents the articular side of the cuff, " $B$ " is bursal, and "C" denotes a complete tear connecting the two surfaces [4].

Snyder classification: Of complete rotator cuff tears:

- Type I: A small, complete tear, $<1 \mathrm{~cm}$.

- Type II: A moderate tear (usually $2-3 \mathrm{~cm}$ ).

- Type III: A large, usually $3-5 \mathrm{~cm}$.

- Type IV: A massive tear [5] . Patients with cuff tears usually have a history of recurrent episodes of shoulder "tendinitis" or "bursitis" [6].

Gouttallier's classification [7] identifies five stages of fatty infiltration:

- Stage 0-normal muscle.

- Stage 1-some fatty streaks.

- Stage 2-less than 50\% fatty muscle infiltration.

- Stage $3-50 \%$ fatty muscle infiltration.

- Stage 4-greater than $50 \%$ fatty muscle infiltration.

For years open surgical repair was the standard which now greatly replaced by mini-open and arthroscopic so we made a comparison between mini-open and arthroscopic rotator cuff repair and assessment was according to ASES score [8]. Liem et al., 2015 study of 38 patients 19 patients had arthroscopic and 19 patients had mini-open repair (mean follow-up, 25.0 months) reported that ar- 
throscopic repair produced equivalent satisfactory clinical results and tendon integrity compared with mini-open repair. They concluded that early range of motion did not differ significantly at 6 weeks or 3 months post-operatively [9]. Fink Barnes et al., 2017, identified 22 patients treated with miniopen and 128 patients treated with arthroscopic rotator cuff repair mean follow-up was two years (1 to 5) outcome was assessed using (ASES) which were higher in the mini-open group (mean ASES score 91.0 in mini-open group; mean 82.70 in the arthroscopic group [10]

\section{Patients and Methods}

We made prospective analysis of 30 patients with rotator cuff tears 15 cases were done arthroscopically and 15 cases done mini-open. The first case was managed in September 2014 and the last in June, 2016. All the cases were managed in ElKasr Al-Ainy and Police-Hospital Agouza. The follow-up period was 6 months. The patients were evaluated using the American Shoulder \& Elbow Society score (ASES), both pre-operatively and post-operatively. Average age for arthroscopic was 52.5 (range, 31-70 years) and 54.4 (42-66 years) for mini-open group. 8 male patients $(53.3 \%)$ and 7 female patients $(64.7 \%)$ in arthroscopic group, 10 males $(66.7 \%), 5$ females $(33.3 \%)$ in mini-open group. 7 patients in arthroscopic group with history of trauma and 8 patients without history of trauma, in mini-open group one patient with history of trauma and 14 patients without. 2 patients were small size tear (less than $1 \mathrm{~cm}$ ), 9 patients were medium size tear $(1-3 \mathrm{~cm}), 4$ patients were large tear $(3-5 \mathrm{~cm})$, no massive tear (more than $5 \mathrm{~cm}$ ) in arthroscopic group.

3 patients were small size tear (less than $1 \mathrm{~cm}$ ), 9 patients were medium size tear $(1-3 \mathrm{~cm})$, and 3 patients were large tear $(3-5 \mathrm{~cm})$, no massive tear (more than $5 \mathrm{~cm}$ ) in mini-open group. Surgical treatment was done after 3 months of failed conservative treatment which includes NSAIDS medication, subacromial steroid injection and physiotherapy.

\section{Surgical technique:}

General anesthesia, beach chair position for all patients in both groups, in arthroscopic group standard arthroscopic portals (posteromedial, posterolateral, lateral, anterolateral, midglenoid portals) was done in all case evaluations of glenohumeral joint for any lesion, tear identification, intraarticular release of adhesions then the arthroscope was introduced into subacromial space bursectomy was done in all cases, tear edge debridement, greater tuberosity debridement for bleeding surface, acromioplasty was done when indicated. Repair done by suture anchors for all cases, 8 cases were repaired by single-row Fig. (1) technique and 7 cases were done by double-row technique Fig. (2) 4 cases of double-row needed side to side repair. In mini-open technique a 5-cm longitudinal skin incision from the tip of the acromion down the lateral aspect of the arm was done, the raphe between the anterior and middle deltoid was identified and spitted from a point $5 \mathrm{~cm}$ or less distal to the acromial border (to avoid axillary nerve injury) toward the antero-lateral acromion Fig. (3), bursectomy, tendon debridement, acromioplasty by removal of anterolateral portion of acromion repair done by suture anchors 10 cases done by singlerow and and 5 cases by double row.

Post-operative management: Patients were placed in arm sling early passive ROM for 3 weeks active assisted exercise after 3 weeks active ROM and strengthening exercise after 6 weeks.

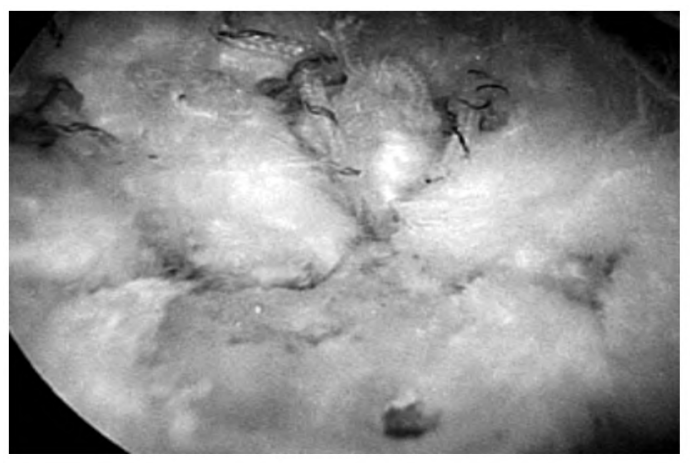

Fig. (1): Single row repair.

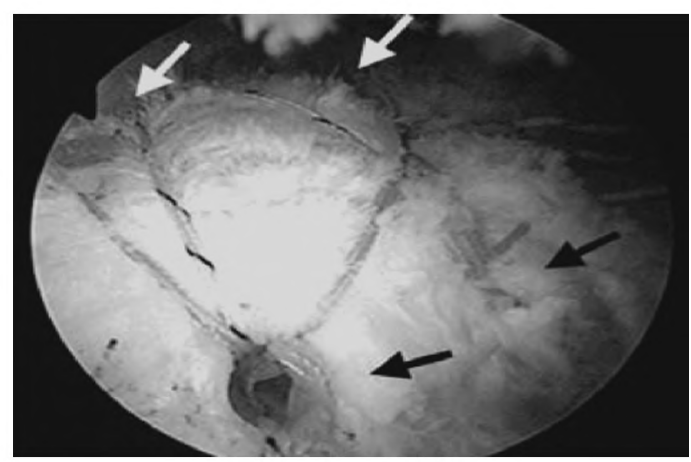

Fig. (2): Double row repair.

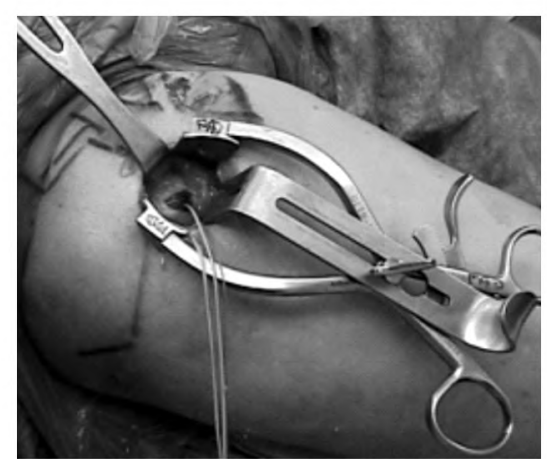

Fig. (3): Mini-open repair. 


\section{Results}

Mean pain score 3 months postoperative for arthroscopic group is (1.73) ranging between (0$4)$, while for mini-open group mean is (4.6) ranging between (3-7) with statistically significant $p$-value $=$ (0.000). Mean pain score 6 months post-operative for arthroscopic group is $(0.27)$ ranging between $(0-3)$, while for mini-open group mean is (1.4) ranging between ( $0-7)$ with statistically insignificant $p$-value $=(0.057)$. The mean total activity score after 3 months for arthroscopic group is (22.93) ranging between (5-27), while it is (13.4) ranging between (3-25) for the mini-open group with statistically significant $p$-value $=(0.000)$. The mean total activity score after 6 months for arthroscopic group is (26.47) ranging between (8-30), while it is (25.6) ranging between (6-29) for the mini-open group with statistically insignificant $p$-value $=(0.7)$. Results for total score of ASES (100 points) was as the following:

-ASES scores 91-100 points for arthroscopic group 13 patients $(86.6 \%)$ and for mini-open group 12 patients $(80 \%)$.

- ASES scores 60-90 points for arthroscopic group one patient (6.66) and for mini-open group 2 patients $(13.33 \%)$.
- ASES scores $<60$ points for arthroscopic group one patient $(6.6 \%)$ and for mini-open group 1 patient $(6.66 \%)$.

\section{Discussion}

Our result is different from Kasten et al., 2011 randomized study of 34 patients 17 patients underwent arthroscopic and 17 underwent mini-open repair reported that VAS pain scores were similar in their arthroscopic and mini-open repair groups for the first 3 weeks postoperatively but that the mini-open repair group had less pain from weeks 4 through 8 . Less use of analgesics was observed during the first postoperative week in their arthroscopic repair group, indicating less pain, but VAS pain scores were higher in weeks 4 through 8 compared with the mini-open repair group [11] regarding total ASES score our study is different from Fink Barnes et al., 2017, identified 22 patients treated with mini-open and 128 patients treated with arthroscopic rotator cuff repair mean followup was two years ( 1 to 5 ) outcome was assessed using (ASES) which were higher in the mini-open group (mean ASES score 91.0 in mini-open group; mean 82.70 in the arthroscopic group [10]. Regarding our complication in comparison to other studies this table summarizes the comparison.

Comparison table between our study and other studies.

\begin{tabular}{|c|c|c|c|c|c|c|}
\hline & \multirow{2}{*}{$\begin{array}{l}\text { Youm et al., } \\
2011 \text { [12] }\end{array}$} & \multirow{2}{*}{$\begin{array}{l}\text { Jung et al., } \\
2012 \text { [13] }\end{array}$} & \multirow{2}{*}{$\begin{array}{l}\text { Kasten et al., } \\
2011 \text { [11] }\end{array}$} & \multirow{2}{*}{$\begin{array}{c}\text { Fink Barnes et al., } \\
2017[\mathbf{1 0}]\end{array}$} & \multicolumn{2}{|c|}{ The results of this study } \\
\hline & & & & & Group A & Group B \\
\hline - Number of cases & -63 & -128 & -34 & -150 & - 15 treateadby & -15 \\
\hline $\begin{array}{l}\text { - Mean ASES score } \\
\text { post-operatively }\end{array}$ & $\begin{array}{l}-91.1 \text { for the } \\
\text { arthroscopic } \\
\text { group and } 90.2 \\
\text { for the mini-open } \\
\text { group }\end{array}$ & - Not mentioned & $\begin{array}{c}-62.6 \text { for group A } \\
67 \text { for group B }\end{array}$ & $\begin{array}{c}-82.70 \text { in group A } \\
91.0 \text { in groupB }\end{array}$ & -92.6 & -91.2 \\
\hline \multicolumn{7}{|c|}{ - Complications: } \\
\hline - Wound infection & $\begin{array}{l}-0 \text { in group A } \\
-1(3.3 \%) \text { for } \\
\text { group B }\end{array}$ & $\begin{array}{l}-1(1.5 \%) \text { for } \\
\text { group A no cases } \\
\text { for group B }\end{array}$ & -0 & - Not mentioed & -0 & $-2(13 \%)$ \\
\hline - Stiffness & - Not mentioned & $\begin{array}{l}\text { - No for both } \\
\text { groups }\end{array}$ & -0 & - Not mentioned & $-2(13 \%)$ & $-3(20 \%)$ \\
\hline
\end{tabular}

\section{Conclusion:}

Mini-open and arthroscopic rotator cuff repair both improved the function of patients who failed non-operative treatment, but there is no statistical or clinically important difference in function between the two surgical approaches according to ASES score. The arthroscopic procedure decreased postoperative pain, faster regains normal ROM and quicker return to function and in turn early return to work compared to mini-open procedure. The arthroscopic procedure better in addressing intra-articular and other associated problems than mini-open technique.

\section{References}

1- DERANLOT J., HERISSON O., NOURISSAT G., et al.: Arthroscopic subacromial spacer implantation in patients with massive irreparable rotator cuff tears: Clinical and 
radiographic results of 39 retrospectives cases. Arthroscopy, Vol. 33: pp. 1639-44, 2017.

2- VAVKEN P., SADOGHI P., PALMER M., et al.: Plateletrich plasma reduces retear rates after arthroscopic repair of small-and medium-sized rotator cuff tears but is not cost-effective Am. J. Sports Med., Vol. 43: pp. 3071-6, 2015.

3- YOON J.P., CHUNG S.W., KIM J.Y., et al.: Outcomes of combined bone marrow stimulation and patch augmentation for massive rotator cuff tears Am. J. Sports Med., Vol. 44: pp. 963-97, 2016

4- HABERMEYER P., KRIETER C., TANG K.I., LICHTENBERG S. and MAGOSH P.: A new arthroscopic classifi cation of articular-sided supraspinatus foot print lesions: A prospective comparison with Snyder's and Elmman's classification. J. Shoulder Elbow Surg., Vol. 17: pp. 909-13, 2014.

5- BERDUSCO R., TRANTALIS J.N., NELSON A.A., et al.: Arthroscopic repair of massive, contracted, immobile tears using interval slides: Clinical and MRI structural follow-up Knee Surg. Sports Traumatol. Arthrosc., Vol. 23: pp. 502, 2015.

6- M. WERNER BAND and COLLIN P.: Correlation between glenoid inclination and critical shoulder angle: A radiographic and computed tomography study. J. Shoulder Elbow Surg., Vol. 24 (12): pp. 1948-53, 2015.

7- LEE C.S., DAVIS S.M., DOREMUS B., KOUK S. and STETSON W.B.: Interobserver agreement in the classification of partial-thickness rotator cuff tears using the snyder classification system. Orthop. J. Sports Med., Vol. 4: pp. 9, 2016.

8- FELLER J.A.: MRI Arthrography updates. Radiology J., pp.: 230-5, 2016.

9- LIEM D., BARTL C., LICHTENBERG S. and MAGOSCH PAND HABERMEYER P.: Clinical out come and tendonintegrity of arthroscopic versus mini-open supraspinatus tendon repair: A magnetic resonance imagingcontrolledmatched-pair nalysis. Arthroscopy, Vol. 23 (5): pp. 514-21, 2015.

10- BARNES L.A., KIM H.M., BUZA J., AHMAD C.S., BIGLIANI L.U. and LEVINE W.N.: Satisfaction, function and repair integrity after arthroscopic versus miniopen rotatorcuff repair; Journal bone and joint, Vol. 99-B: pp. 245-9, 2017.

11- KASTEN P., KEIL C., GRIESER T., RAISS P. and STREICH N.: Prospective randomized comparison of arthroscopic versus mini-open rotator cuff repair of the supraspinatus tendon. Int. Orthop., Vol. 35: pp. 1663-70, 2011.

12- YOUM T., MURRAY D.H., KUBIAK E.N., ROKITO A.S. and ZUCKERMAN J.D.: Arthroscopic versus mini open rotator cuff repair: Acomparison of clinical outcomes and patient satisfaction, J. Shoulder. ElbowSurg., Vol. 14: pp. 455-9, 2011.

13-JUNG G.H., LEE Y.K. and SHIN H.K.: Early postoperative outcomes between arthroscopic and mini-open repair for rotator cuff tears. Orthopedics online, Vol. 35: pp. 347, 2012.

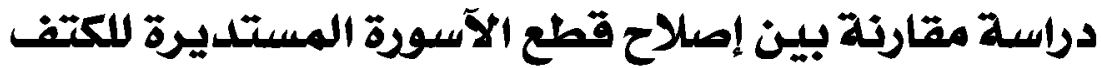

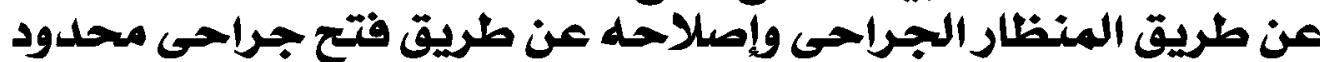

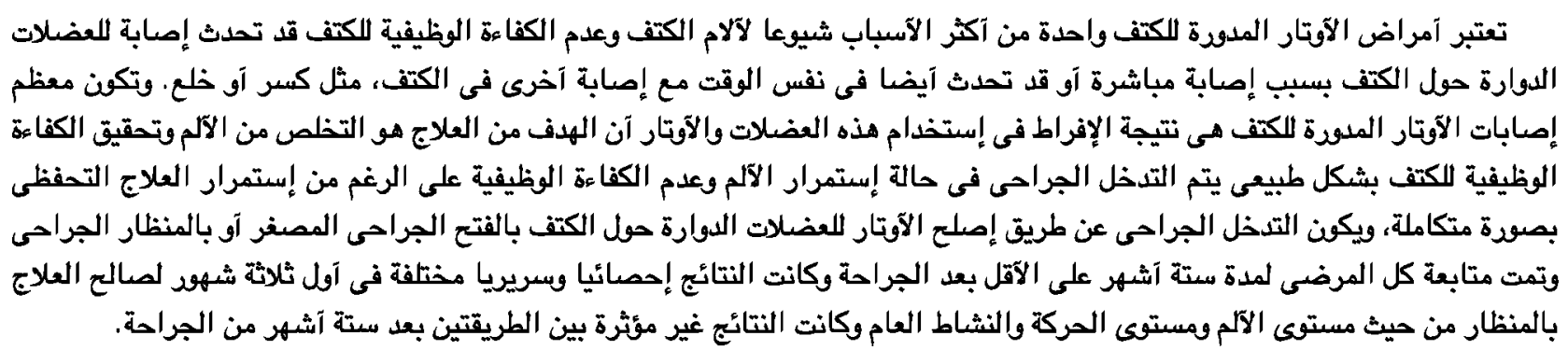

